\title{
A Novel Design of Iron Dominated Superconducting Multipole Magnets with Circular Coils
}

\author{
Vladimir Kashikhin
}

\begin{abstract}
Linear accelerators based on superconducting magnet technology use a large number of relatively weak superconducting quadrupoles. In this case an iron dominated quadrupole is the most cost effective solution. The field quality in this magnet is defined by iron poles; the magnet air gap is minimal as are coil ampere-turns. Nevertheless, it has long racetrack type coils, which must be rigid and fixed by a mechanical structure to provide the needed mechanical stability. The novel concept of using circular superconducting coils in such a quadrupole type is described, with a discussion of quadrupole parameters, and results of 3D magnetic designs. Variants of short and long sectional quadrupoles and multipoles are presented.
\end{abstract}

Index Terms- Accelerator magnets, Superconducting magnets, Magnetic fields, Coils, Iron.

\section{INTRODUCTION}

S EVERAL Linear Accelerators based on $\checkmark$ superconducting technology now are under design and construction [1-3]. These machines use various superconducting magnets to provide particle beam steering and focusing. The main beam focusing element is a superconducting quadrupole mounted inside a cryomodule between SCRF cavities [4-6].

\section{QUADRUPOlE MAGNeT CONCEPT}

There are two types of superconducting quadrupoles used in Linear Accelerators with shell or racetrack coils. The magnet for TESLA Test Facility was based on shell type coils [4]. For ILC [1], XFEL [2], and Project-X [3] relatively weak quadrupoles are based on racetrack type coils where the magnetic field is shaped by iron poles [4-8]. Because the beam size in these accelerators is very small, this approach is more efficient from a technological point of view. Also, superconductor magnetization effects in such magnets are lower [5], [8].

The main idea of the proposed magnet is to use circular coils to generate a quadrupole field. Such approach is used in special electrical machines with "nose type poles". The magnetic flux in this case should be directed around this coil to magnet poles having a quadrupole configuration as shown

Manuscript received 18 October 2009. This work was supported by the U.S. Department of Energy.

V. Kashikhin is with the Fermi National Accelerator Laboratory, Batavia, IL 60510 USA (phone: 630-840-2899; fax: 630-840-3369; e-mail: kash@fnal.gov). in Fig. 1. This geometry was proposed by I.F. Malyshev for a room temperature quadrupole. But because of large room temperature coil cross-section there was not enough space for efficient flux returns which were saturated at very low quadrupole field gradients.

This approach was modified for superconducting short and long multipole magnets with different apertures, and is discussed below.

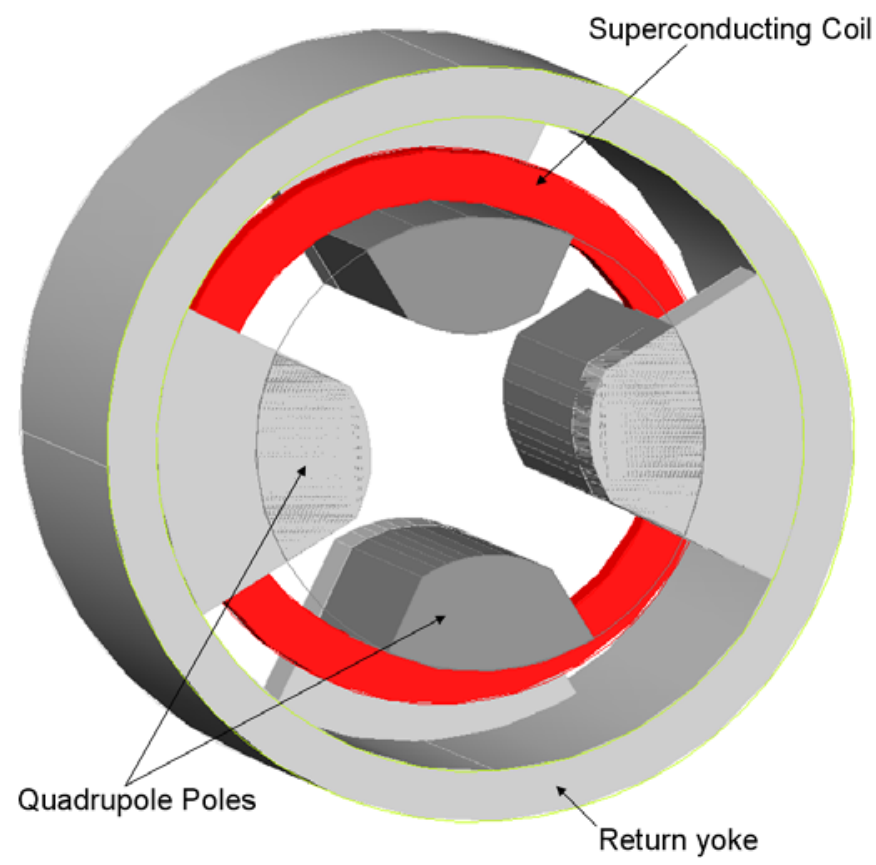

Fig. 1. Iron dominated quadrupole with circular coil.

\section{QuAdRUPOle MAGNeTIC DESIGN}

The quadrupole magnet specification depends on the accelerator parameters: type of particles, particle energy, and needed aperture. It is supposed for ILC, Project-X, and XFEL to have an $80 \mathrm{~mm}$ aperture diameter. The possibility was discussed to halve the aperture, to make the quadrupole more compact, and reduce the fringe fields in the area of SCRF cavities. Quadrupole integrated gradients change with particle energy over a large range: from $2 \mathrm{~T}$ up to $5.6 \mathrm{~T}$ in XFEL, and up to $36 \mathrm{~T}$ in ILC. This leaves enough design space for the compact low gradient quadrupole approach which could be used at low energies. The investigated quadrupole peak gradient is limited by the iron yoke saturation. The total pole magnetic flux goes through the relatively narrow cross-section 
of pole flux return and proper geometry should be chosen to reduce the effect of this limitation. The small cross-section of superconducting coil allows to increase the thickness of flux returns and achieve high field gradients. Another possible solution is to use in these areas a high saturation material like Permendur.

\section{A. Single Coil Quadrupole}

The investigated single coil quadrupole geometry is shown in Fig. 2. The quadrupole performance was investigated using OPERA 3D code [9]. The magnet parameters are shown in Table 1.

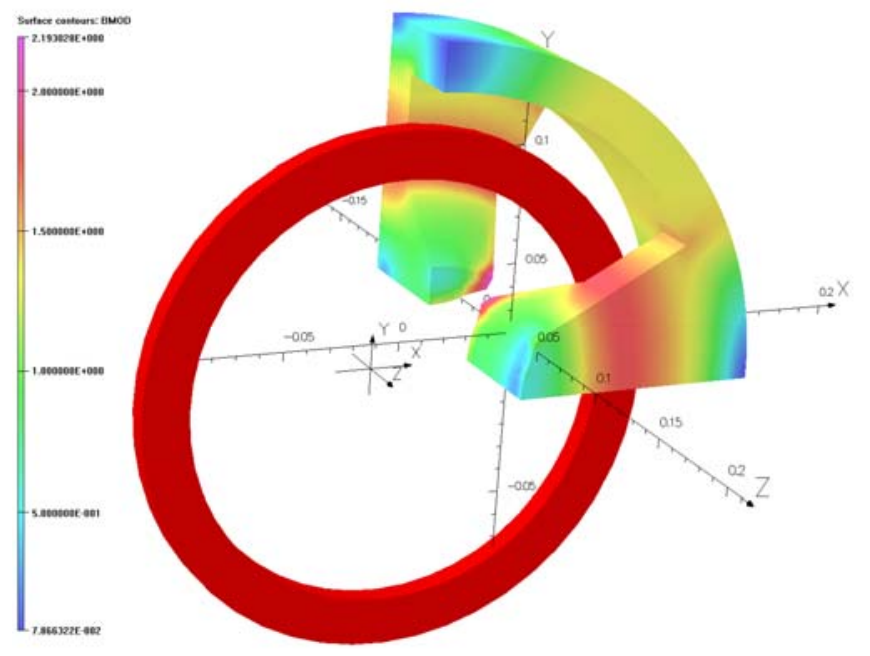

Fig. 2. Quarter of single coil quadrupole geometry with $90 \mathrm{~mm}$ aperture diameter and flux density distribution in the iron yoke.

\begin{tabular}{cccc} 
TABLE I SINGLE COIL QUADRUPOL PARAMETERS \\
\hline Parameter & Units & $\begin{array}{c}\text { Aperture } \\
\mathbf{8 0} \mathbf{~ m m}\end{array}$ & $\begin{array}{c}\text { Aperture } \\
\mathbf{4 5} \mathbf{~ m m}\end{array}$ \\
\hline Quadrupole pole tip diameter & $\mathrm{mm}$ & 90 & 45 \\
Peak field gradient & $\mathrm{T} / \mathrm{m}$ & 5.54 & 10.2 \\
Quadrupole length & $\mathrm{mm}$ & 50 & 50 \\
Coil ampere-turns & $\mathrm{kA}$ & 8 & 4 \\
Peak field in the coil & $\mathrm{T}$ & 0.4 & 0.3 \\
Iron yoke peak field & $\mathrm{T}$ & 2.2 & 2.12 \\
Integrated gradient & $\mathrm{T}$ & 0.43 & 0.71 \\
Effective length & $\mathrm{mm}$ & 78 & 70 \\
Outer yoke diameter & $\mathrm{mm}$ & 300 & 150 \\
\hline
\end{tabular}

One can see strong iron saturation in the flux return legs. At two times smaller aperture and coil ampere-turns the achievable field gradient is two times larger. This effect is caused by smaller aperture and proportionally higher flux concentration between poles. It should be noted that the single coil magnet has a low total integrated gradient because of its short length. It is possible to increase the integrated field by increasing the magnet pole length with proportional increase of flux return cross-section.

One of the main issues for ILC quadrupoles is the superconductor magnetization effect [5], [8]. In this design the coil is shielded by the iron and field distortions caused by magnetization effects should be low.

\section{B. Double Coil Quadrupole}

In many cases for a single coil quadrupole there is not enough quadrupole integrated field to generate a specified strength. Besides, the single coil magnet produces some relatively low solenoidal Bz-field component. It could be used for the beam focusing but also could be compensated by adding a second magnet having the same configuration but reflected relative to the first one in the $\mathrm{X}-\mathrm{Y}$ plane as shown in Fig. 3. The current in this second magnet should have the opposite direction to the current in the first magnet. This double coil quadrupole shown in Fig. 3.

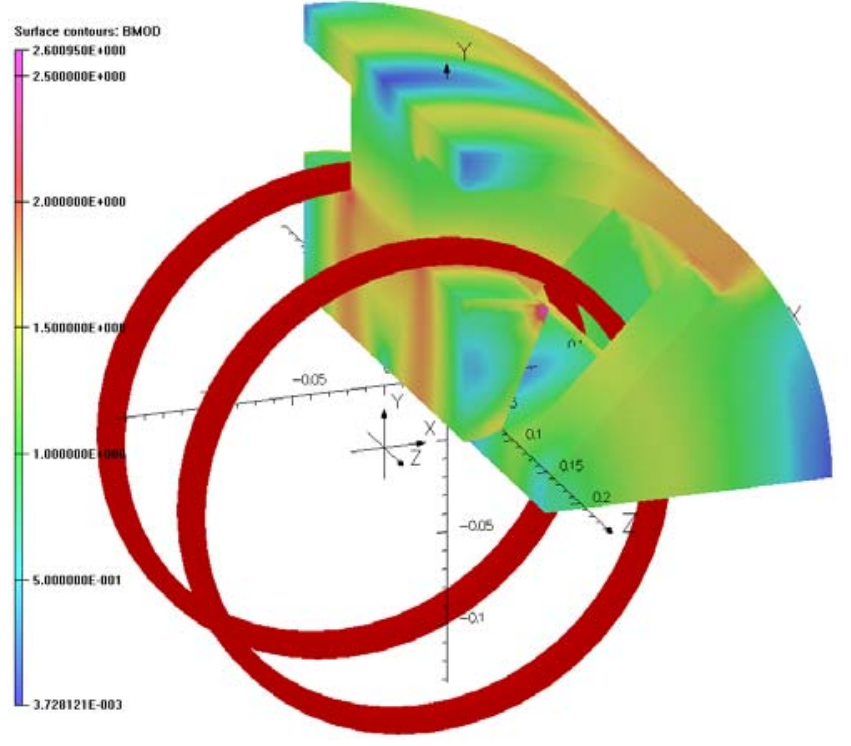

Fig. 3. Quarter of double coil quadrupole geometry and flux density distribution in the iron yoke.

TABLE II. DOUble COIL QUAdRUPOLE PARAMETERS

\begin{tabular}{cccc}
\hline \hline Parameter & Units & $\begin{array}{c}\text { Aperture } \\
\mathbf{8 0 ~} \mathbf{~ m m}\end{array}$ & $\begin{array}{c}\text { Aperture } \\
\mathbf{4 5} \mathbf{~ m m}\end{array}$ \\
\hline Quadrupole pole tip diameter & $\mathrm{mm}$ & 90 & 45 \\
Peak field gradient & $\mathrm{T} / \mathrm{m}$ & 7.1 & 12.6 \\
Quadrupole length & $\mathrm{mm}$ & 240 & 240 \\
Coil ampere-turns & $\mathrm{kA}$ & 12 & 6 \\
Peak field in the coil & $\mathrm{T}$ & 0.3 & 0.42 \\
Iron yoke peak field & $\mathrm{T}$ & 2.6 & 2.27 \\
Integrated gradient & $\mathrm{T}$ & 2.02 & 2.97 \\
Effective length & $\mathrm{mm}$ & 285 & 236 \\
Outer yoke diameter & $\mathrm{mm}$ & 400 & 200 \\
\hline \hline
\end{tabular}

One can see that the double coil quadrupole is capable of generating $2 \mathrm{~T}-3 \mathrm{~T}$ of integrated field gradient in a length of $240 \mathrm{~mm}$. 


\section{Long Quadrupoles}

Integrated field gradients larger than $3 \mathrm{~T}$ could be obtained by several replications of the double coil design as shown in Fig. 4. The quadrupole parameters are close to the double coil design. But two times longer magnet produces correspondingly two times larger integrated gradients.

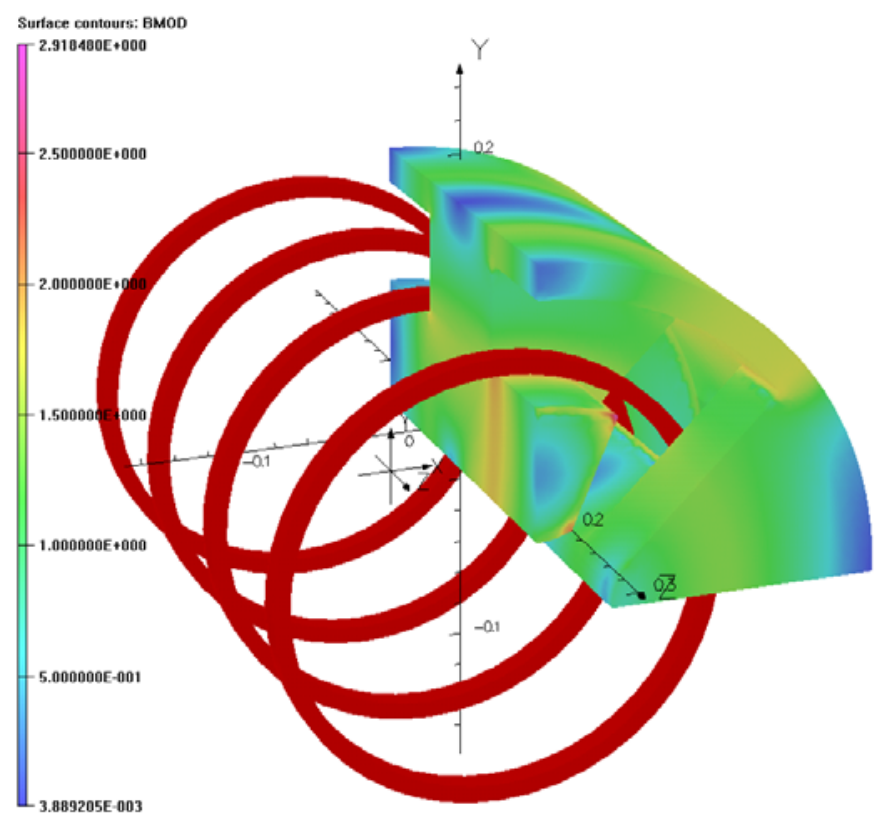

Fig. 4. Quarter of four coil quadrupole geometry and flux density distribution in the iron yoke. Half length of yoke is shown.

\section{TABLE III. FOUR COIL QUADRUPOLE PARAMETERS}

\begin{tabular}{cccc}
\hline \hline Parameter & Units & $\begin{array}{c}\text { Aperture } \\
\mathbf{8 0} \mathbf{~ m m}\end{array}$ & $\begin{array}{c}\text { Aperture } \\
\mathbf{4 5} \mathbf{~ m m}\end{array}$ \\
\hline Quadrupole pole tip diameter & $\mathrm{mm}$ & 90 & 45 \\
Peak field gradient & $\mathrm{T} / \mathrm{m}$ & 7.43 & 12.36 \\
Quadrupole length & $\mathrm{mm}$ & 480 & 480 \\
Coil ampere-turns & $\mathrm{kA}$ & 12 & 6 \\
Peak field in the coil & $\mathrm{T}$ & 0.31 & 0.24 \\
Iron yoke peak field & $\mathrm{T}$ & 2.92 & 2.56 \\
Integrated gradient & $\mathrm{T}$ & 3.84 & 5.87 \\
Effective length & $\mathrm{mm}$ & 517 & 475 \\
Outer yoke diameter & $\mathrm{mm}$ & 400 & 200 \\
\hline \hline
\end{tabular}

The long quadrupole field could be decreased by excluding some coils from electrical circuit during assembly or by using superconducting switches. For example, if end coils (See Fig. 4) are not powered the integrated gradient for a 90 $\mathrm{mm}$ aperture quadrupole drops from $3.84 \mathrm{~T}$ to $1.07 \mathrm{~T}$. This is good for a front end of accelerator where the particle energy rises quickly.

\section{Quadrupole Gradient Distribution}

The main parameter of a quadrupole is the magnetic field gradient. For short magnets a large portion of the integrated gradient is generated by fringe fields. Fig. 5 shows the quadrupole gradient distributions for various magnet lengths. There is some difference in the form of gradient distributions for $\mathrm{X}-\mathrm{Z}$ and $\mathrm{Y}-\mathrm{Z}$ planes caused by the iron saturation in different pole areas. Nevertheless, the same total flux passes the magnet gap (flux conservation law) and it equalizes integrated gradients for both planes and any angular plane position. The high homogeneity of integrated fields could be obtained by proper pole surface shimming.

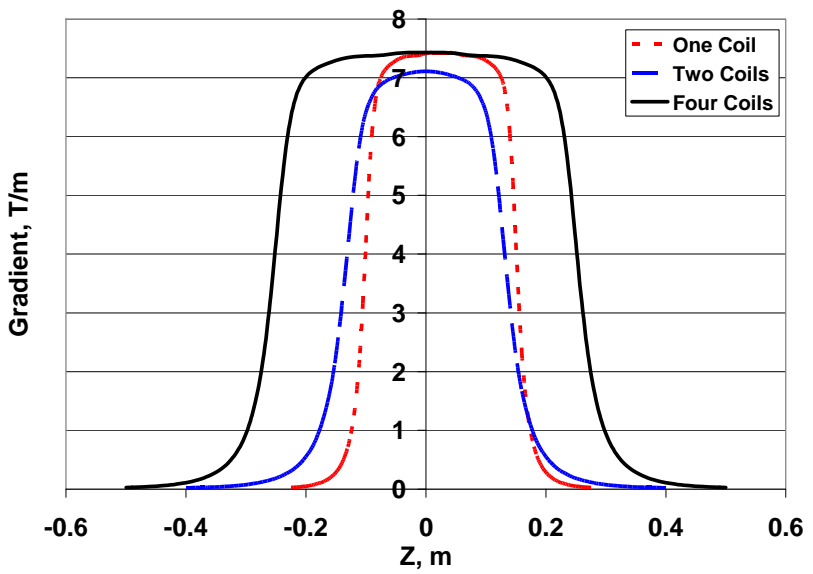

Fig. 5. Quadrupole (90 $\mathrm{mm}$ bore) gradient distribution for the single coil, the two coils, and the four coils versions .

Field gradients have a sharp drop at both quadrupole ends. As a result the effective magnetic field length is close to the magnet pole length.

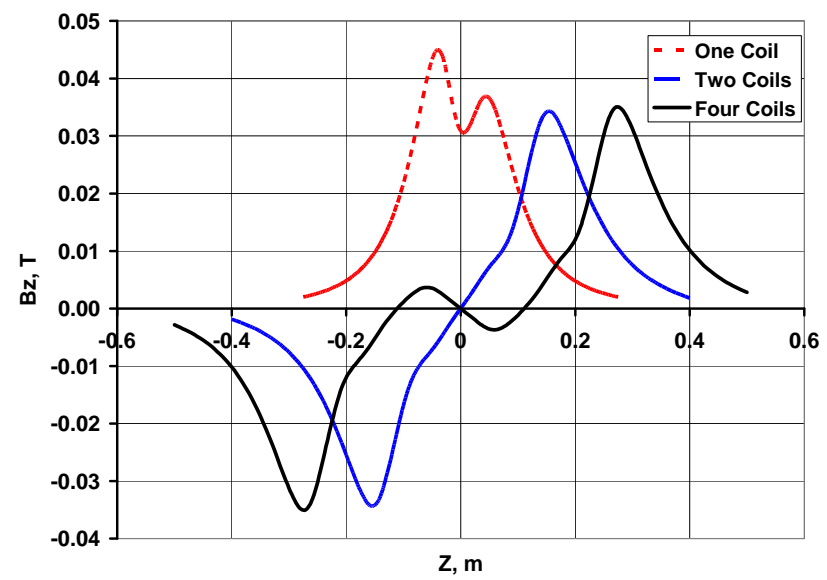

Fig. 6. Quadrupole (90 $\mathrm{mm}$ bore) $\mathrm{Bz}$ - field distribution for the single coil, two coils, and the four coils versions.

The solenoidal field component $\mathrm{Bz}$ (See Fig. 6) is substantial for the single coil design. But the integrated $\mathrm{Bz}$ field is fully eliminated by opposite current directions in the neighboring coils.

\section{Quadrupole Mechanical Design}

The magnet mechanical design includes two main assemblies: circular coils and the iron yoke. The section of the yoke is assembled from low carbon steel pieces. There are two ways of machining yoke parts and assembly with the coil. One way is to cut the iron yoke assembly under $45^{\circ}$. Two identical iron parts are machined from the AISI 1006 - AISI 1010 low carbon steel and bolted to each other during final assembly with the coil. The high precision of pole profiles 
could be obtained by final EDM machining of these surfaces when parts are bolted to each other. All other yoke surfaces have low tolerances, which allows inexpensive machining. Another way is to split one coil yoke section by cutting the outer flux return ring in the $\mathrm{X}-\mathrm{Y}$ plane. This way allows axial parts assembly with the coil.

The magnet coils are wound into a circular aluminum bobbin and then impregnated with epoxy in vacuum. Coils are wound using $0.5 \mathrm{~mm}$ diameter enamel-insulated $\mathrm{NbTi}$ superconductor capable carry up to $200 \mathrm{~A}$. So, the coil number of turns is defined by the design current which is usually less than or equal to $100 \mathrm{~A}$.

The superconducting magnet performance in general is defined by coil mechanical stability. The solenoids have the best configuration to protect wires from displacements. Lorentz forces in these coils are intercepted by the coil bobbin and are transferred in to hoop stresses. This is very useful for coils with hundreds of turns to avoid long coil training and quenches because of epoxy cracking.

\section{Multipole magnets}

The proposed quadrupole approach could be extended to dipole and multipole magnets. Most multipole magnets are used as correctors and have relaxed parameters related to the field strength and quality. It is very attractive to use only one or two circular coils to generate sextupole or octupole fields. Fig. 6 shows a sextupole magnet with two coils and in Table 4 the double coil sextupole parameters are presented.

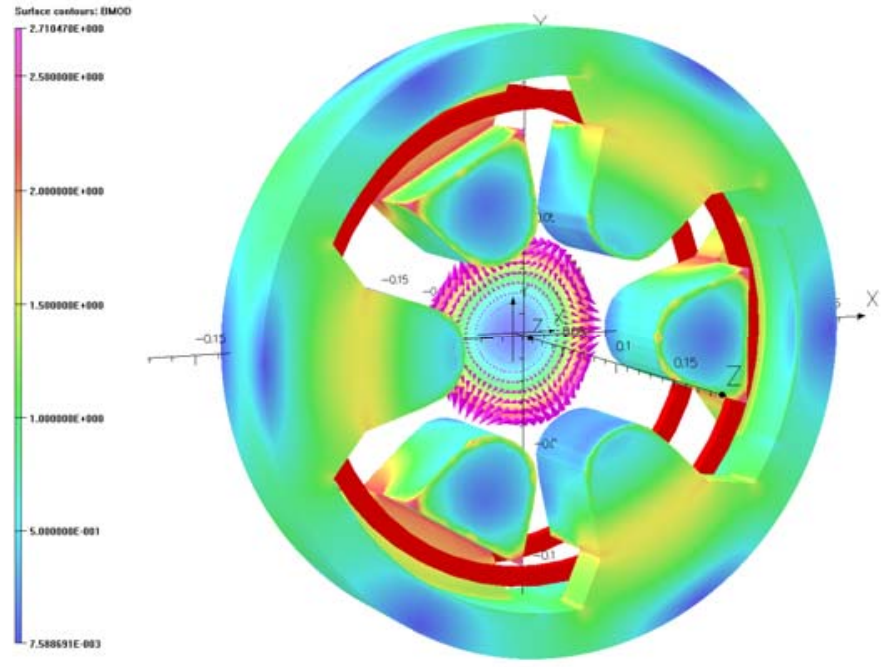

Fig. 6. Double coil sextupole geometry and flux density distribution in the iron yoke. Half length of yoke and vectors $\mathbf{B}$ in the aperture are shown.

TABLE IV. Two Coll SeXtupole Parameters

\begin{tabular}{cccc}
\hline \hline Parameter & Units & $\begin{array}{c}\text { Aperture } \\
\mathbf{8 0} \mathbf{~ m m}\end{array}$ & $\begin{array}{c}\text { Aperture } \\
\mathbf{4 5} \mathbf{~ m m}\end{array}$ \\
\hline Sextupole pole tip diameter & $\mathrm{mm}$ & 90 & 45 \\
Peak field gradient & $\mathrm{T} / \mathrm{m}^{2}$ & 102.8 & 388.5 \\
Sextupole length & $\mathrm{mm}$ & 100 & 100 \\
Coil ampere-turns & $\mathrm{kA}$ & 5 & 2.5
\end{tabular}

\begin{tabular}{cccc} 
Peak field in the coil & $\mathrm{T}$ & 0.4 & 0.24 \\
Iron yoke peak field & $\mathrm{T}$ & 2.71 & 2.54 \\
Integrated gradient & $\mathrm{T} / \mathrm{m}$ & 11.7 & 44.1 \\
Effective length & $\mathrm{mm}$ & 114 & 113 \\
Outer yoke diameter & $\mathrm{mm}$ & 270 & 135 \\
\hline
\end{tabular}

\section{CONCLUSION}

The proposed magnets have the following advantages:

- Simple and self-supporting circular coil configuration.

- Low coil field allows to use HTS superconductor.

- Large coil diameter allows to wind coil using react and wind technology for HTS.

- Single coil for the section of multipole correctors.

- Long magnet design with possibility to turn on or off identical magnet sections.

- Low cost of manufacturing.

In the paper discussed novel magnet configurations with circular coils. The geometry of magnets was not optimized to obtain high field gradients and a good field quality in a large aperture. This should be made for magnets with well-known specifications which is different for various accelerators. The main goal of this paper just to show the way to make magnets with simple circular coils.

Nevertheless, it should be noted that this type of magnet is suitable for low integrated field applications like front end of linear accelerators or low energy beam transport lines. This approach was investigated as an option for one of FNAL NML [10] Cryomodule quadrupole.

\section{REFERENCES}

[1] "International Linear Collider Reference Design Report", http://www.linearcollider.org/cms/?pid=1000025.

[2] "The European X-Ray Free-Electron Laser. Technical Design Report", DESY 2006-097, July 2007.

http://xfel.desy.de/localfsExplorer_read?currentPath=/afs/desy.de/group/ xfel/wof/EPT/TDR/XFEL-TDR-final.pdf

[3] "Project-X Draft Report from the ICD-2 Research Program", FNAL, October 2, 2009. http://www.fnal.gov/pub/projectx/pdfs/ICD 2_Research_Program_Task_Force_v6.pdf

[4] F. Toral,_et. al., "Fabrication and Testing of a Combined Superconducting Magnet for the TESLA Test Facility", IEEE Transactions on Applied Superconductivity, vol. 16. No. 2, June 2006, pp. 231-235.

[5] F. Toral,_et. al., "Magnetization Effects on the Superconducting Combined Magnet Prototype for XFEL"', IEEE Transactions on Applied Superconductivity, vol. 19. No. 3, June 2009, pp. 1136-1140.

[6] A. Koski, R. Bandelmann, S. Wolff, "Superconducting magnet package for the TESLA test facility”, IEEE Transactions on Magnetics, vol. 32, No. 4, July 1996, pp. 2155-2158.

[7] V.S. Kashikhin, et. al., “ Design and Manufacturing Main Linac Superconducting Quadrupole for ILC at Fermilab"', IEEE Transactions on Applied Superconductivity, vol. 18. Issue 2, June 2008, pp. 155-158.

[8] [6] V. S. Kashikhin , et. al., "Test Results of a Superconducting Quadrupole Model Designed for Linear Accelerator Applications", IEEE Transactions on Applied Superconductivity, vol. 19. Issue 3, Part 2, June 2009, pp. 1176-1181.

[9] OPERA 3D TOSCA, Cobham Technical Services, Vector Fields Software, 24 Bankside, Kidlington, Oxford OX5 1JE, England.

[10] "NML- New Muon Lab", Fermilab, 2009. http://apc.fnal.gov/ARDWS/NML.html. 